\title{
Compact Photonic Integrated Phase and Amplitude Controller for Phased-Array Antennas
}

\author{
J. Stulemeijer, F. E. van Vliet, Member, IEEE, K. W. Benoist, D. H. P. Maat, and M. K. Smit, Associate Member, IEEE
}

\begin{abstract}
Feasibility of an extremely compact photonic integrated circuit for controlling phase and amplitude of a 16element phased-array microwave antenna has been demonstrated experimentally.
\end{abstract}

Index Terms-Beam steering, integrated optics, optical phase shifters, optical waveguides, phased-array radar.

\section{INTRODUCTION}

$\mathbf{P}$ HASED-ARRAY antennas have been used since the beginning of the 1970's and are now becoming increasingly important in satellite and mobile communications. A drawback for broad application of phased-array antennas is the large volume and weight of the RF electronic beam-forming network. Photonics holds a great promise for reducing both weight and volume of these networks. Many experiments have been reported based on discrete optical components [1]-[3]. First integrated circuits were realized on lithium niobate, see [4] and [5]. In this paper we report the first experiments on an InP-based integrated beam-forming network with extremely small dimensions $\left(8.5 \times 8 \mathrm{~mm}^{2}\right)$ for controlling phase and amplitude of a 16-element RF phased-array antenna. InP has the advantage that it allows for integration of high extinction electroabsorption modulators and optical amplifiers for compensating on-chip losses in large circuits. Further device size can be much smaller as compared with lithium niobate.

\section{OPERATION PRINCIPLE}

Using a coherent detection scheme phase and amplitude of an optical signal can be directly transferred to a microwave signal by mixing this signal with an optical local oscillator signal. In this way, modulation of phase and amplitude of a microwave signal can be performed using optical phase and amplitude modulators, which provides the module with an almost unlimited bandwidth; phase and amplitude modulation efficiency is flat in a frequency range from a couple of $\mathrm{MHz}$ up to tens of $\mathrm{GHz}$ (only limited by the photo-detector). Fig. 1 illustrates the schematic of the photonic beam control network, which has been realized. The optical chip is shown within the dotted box. The chip has two inputs for two optical signals, one

Manuscript received July 15, 1998; revised October 12, 1998.

J. Stulemeijer and M. K. Smit are with the Department of Information Technology and Systems, TTT Laboratory, Integrated Photonics Group, Delft University of Technology, 2600 GA Delft, The Netherlands.

F. E. van Vliet and K. W. Benoist are with TNO-Physics and Electronics Laboratory, 2509 JG The Hague, The Netherlands.

D. H. P. Maat is with the Delft University of Technology, Department of Applied Physics, 2600 GA Delft, The Netherlands.

Publisher Item Identifier S 1041-1135(99)00377-8.

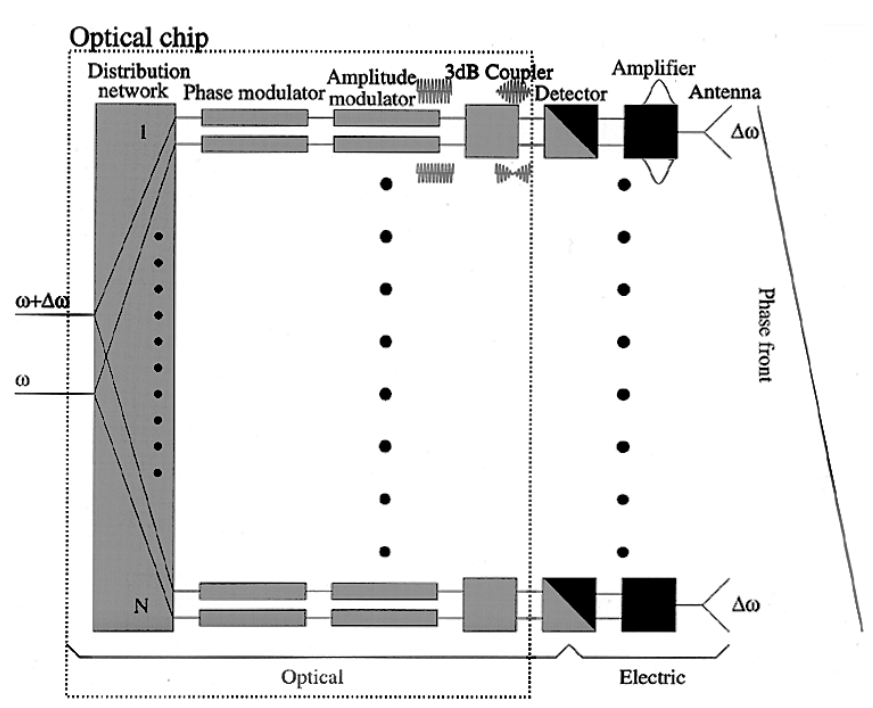

Fig. 1. Schematic view of the optical control of a phased-array antenna.

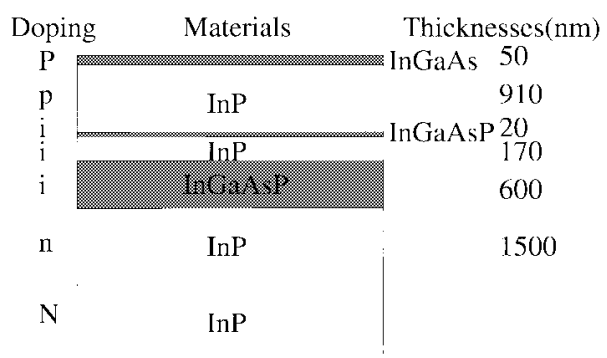

Fig. 2. Layer-stack.

of which is modulated with the radar pulse. The two inputs are fed to two $1 \times 16$ power splitting networks, which are overlapping in such a way that every output of the first network is situated next to an output of the second network. Each pair is connected to a phase and amplitude modulation section, after which the two signals are fed to a 3-dB coupler. The RFsignals are obtained by coupling the signals coming out of the sixteen $3-\mathrm{dB}$ couplers to a series of 16 balanced detector pairs. In the following we will discuss the design and performance of the different elements.

\section{FABRICATION}

We developed our circuit in a MOVPE grown InPInGaAsP-InP double-heterostructure as usual for operation in the long wavelength window $(1550 \mathrm{~nm})$ for optical communication, see Fig. 2. 


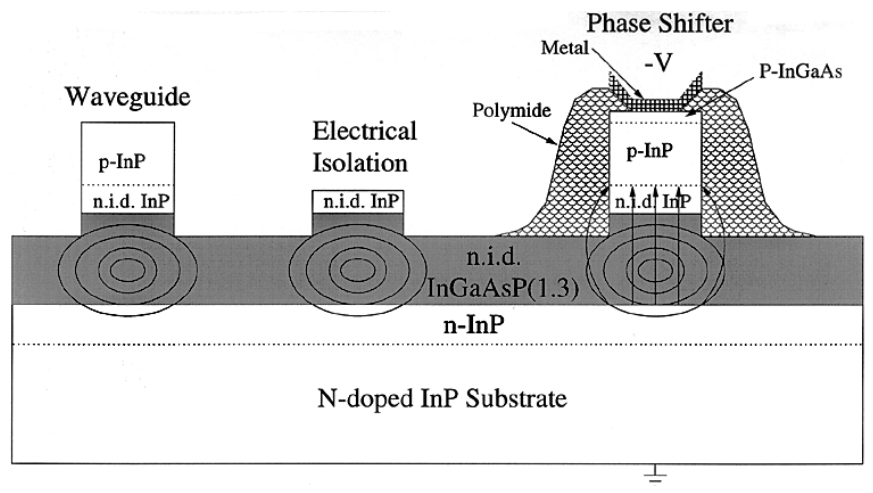

Fig. 3. Waveguides on chip.

First, the InGaAs contact layer is removed everywhere excepts for the contacts on top of the phase/amplitude modulators. This is done using a selective wet chemical etch with photo resist as etch mask. Next a SiN layer is deposited by PECVD and photoresist is spun on top. The waveguide mask is defined in the photoresist by contact lithography. This pattern is transferred to the $\mathrm{SiN}$ by use of a $\mathrm{CHF}_{3}$ plasma in a reactive-ion-etching (RIE). Ridge-type waveguides are etched with an optimized $\mathrm{CH}_{4}-\mathrm{H}_{2}$ process with the SIN as etch mask. When part of the cladding is etched the SiN layer on top of the waveguides in front and behind the phase/amplitude modulators is removed, such that the p-doped part of the cladding will be removed here when the waveguide etch is finished. This prevents short-circuits through the cladding between the different phase/amplitude modulators. Next photosensitive polymide is spun on the chip and a pattern is defined such that the phase/amplitude modulators are covered with polymide with an contact window on top of the ridge. Finally, gold is deposited on the contact openings and a pattern is defined using a liftoff process. The normal waveguide, the waveguide in the electrical isolation region and the waveguide in the phase/amplitude modulator are shown in Fig. 3.

\section{Measurements}

\section{A. Passive Waveguides}

The chip was designed for use with TE polarized light, this does not impose any problems because the input lasers provide linearly polarized light. For interconnection we use 2$\mu \mathrm{m}$-wide waveguides, see Fig. 4(a). The total on chip loss was around $28 \mathrm{~dB}$ with a spread of $1.0 \mathrm{~dB}$ measured for input one to all outputs and input 2 to all outputs. Dummy waveguide crossings, see Fig. 4(c), were introduced to eliminate loss differences between different paths. Power splitters and 3-dB-couplers have been realized by applying multimode interference couplers [6]. These couplers consist of a wide multimode waveguide section as shown in Fig. 4(a), which exhibits self-imaging properties.

The average on chip-loss is composed as follows. The theoretical splitting loss of a $1 \times 16$ splitter is $12 \mathrm{~dB}$ and the losses at a MMI mixer are $3 \mathrm{~dB}$, leaving an excess loss of $13.1 \mathrm{~dB}$. Test structures were included to measure the losses for straight waveguides, bends and waveguide crossings.

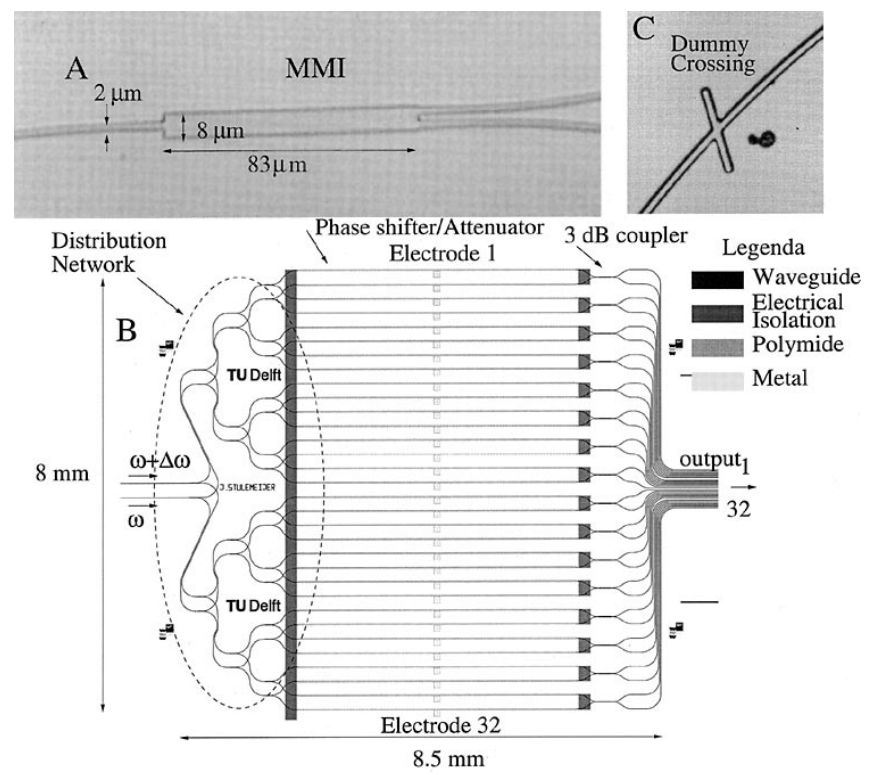

Fig. 4. (a) A MMI power splitter. (b) Mask layout of chip. (c) Dummy crossing to equalize output powers.

Straight waveguides of $2 \mu \mathrm{m}$ width have a loss of $1.4 \mathrm{~dB} / \mathrm{cm}$. Measurements showed a maximum loss of $0.15 \mathrm{~dB} / 90^{\circ}$ for bend waveguides with radius of $250 \mu \mathrm{m}$. The loss was 0.15 $\mathrm{dB}$ for a crossing of waveguides under an angle of $60^{\circ}$. The insertion loss, (without applied voltage) for a phase shifting section including the electrical isolation sections is about $1 \mathrm{~dB}$. Combining the results of the test structures with the layout of the chip we expect the excess loss to be $4.1 \mathrm{~dB}$. Part of the additional $9 \mathrm{~dB}$ is caused by a problem in the processing. Based on previous experiments on $1 \times 16$ splitters [7] we expect that the total excess loss can be below $6 \mathrm{~dB}$.

\section{B. Phase-Modulation Sections}

Phase shifting is accomplished by utilizing refractive index changes due to the electro-optic effect. The doping profile of the chip is chosen in such a way that the InGaAsP waveguiding layer gets depleted when a reverse bias is applied to the waveguide, see Fig. 2. This way the overlap between the electrical field and the optical field is maximal, for further details see [8].

In Fig. 5, it can be seen that more than $700^{\circ}$ of phase shift can be realized with a 4.9-mm-long electrode by applying a negative voltage smaller then $20 \mathrm{~V}$. Taking into account the length of $4.9 \mathrm{~mm}$ of the phase shifting section it follows that the efficiency is $8.2 \%(\mathrm{~V} \mathrm{~mm})$. At values below $-10 \mathrm{~V}$ the insertion loss becomes impractically high, as can be seen from Fig. 6; if the negative voltage is kept within $5 \mathrm{~V}$ the additional loss is kept within $1 \mathrm{~dB}$. With such a voltage a phase shift ranging from $-180^{\circ}$ to $+180^{\circ}$ can be realized by applying the control signal to one or to the opposite electrode.

\section{Amplitude Modulators}

An advantage of the p-i-n-type waveguide phase-modulator is that, with a proper design, it can be used also as an amplitude modulator using the electroabsorption effect which occurs at 


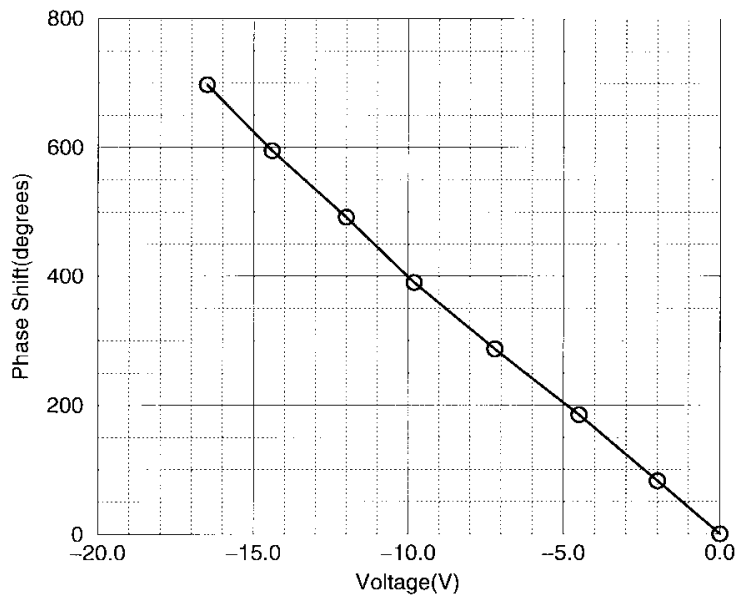

Fig. 5. Phase shift as function of applied voltage.

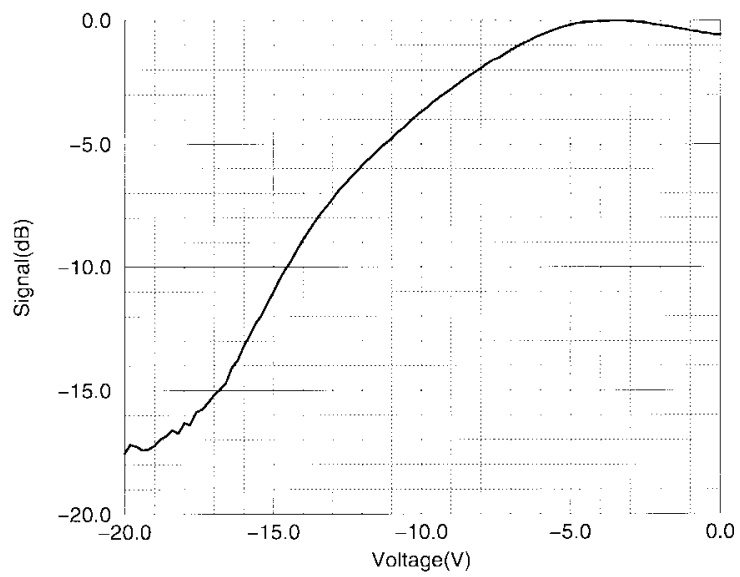

Fig. 6. Attenuation as a function of applied voltage.

higher voltages due to the electrical-field induced shift of the band-edge. The absorption is expected to be sensitive to both temperature and wavelength, so temperaure stabilization will be necessary. The wavelength dependence is no problem because of the small optical badwidths $(\ll 100 \mathrm{GHz} \approx 0.8 \mathrm{~nm})$ required in this application. Fig. 6 shows the attenuation as a function of the voltage, it is seen that an attenuation of over $15 \mathrm{~dB}$ can be achieved with an applied voltage of $-20 \mathrm{~V}$.

After passing the phase shifter/attenuator sections the laser signals are fed to a $2 \times 2 \mathrm{MMI}$ coupler and then fed to a detector pair where they generate an RF-current which is proportional to the product of the optical input signals. In principle, the phase of the RF-signal can be adjusted with one electrode and its amplitude with the other. As the phase and amplitude transfer of the modulator sections are coupled the control signals on both electrodes are not independent but using a proper combination of voltages any amplitude-phase combination of the RF-signal can be addressed.

\section{CONCLusion}

We have realized a distribution network consisting of two $1 \times 16$ power splitters integrated with phase shifting sections on an InP chip with record small dimensions $(8.5 \times 8 \mathrm{~mm})$. The on-chip loss is $28 \pm 1.0 \mathrm{~dB}$. The phase/attenuation sections have a phase dynamic range of more than $360^{\circ}$ and an attenuation dynamic range of almost $20 \mathrm{~dB}$.

\section{ACKNOWLEDGMENT}

The authors would like to thank Phillips Opto-electronics for kindly providing them with two micro-positioners. Further, they thank E. Radius from KPN Research Optical Network Innovation group for the use of one of their EDFA's. The authors would also like to thank F. van Putten from TNO-FEL for assistance during the measurements.

\section{REFERENCES}

[1] H. Zmuda and E. N. Toughlian, Photonic Aspects of Modern Radar, 1st ed. Norwood, MA: Artech House, 1994.

[2] A. J. Seeds, "Application of opto-electronic techniques in phased array antenna beamforming," in Proc. Microwave Photonics Conf., 1997, pp. $15-20$.

[3] J. J. Lee, R. Y. Loo, S. Livingston, V. I. Jones, J. B. Lewis, H. W. Yen, G. L. Tangonan, and M. Wechsberg, "Photonic wideband array antennas," IEEE Trans. Antennas Propagat., vol. 43, pp. 966-982, Sept. 1995.

[4] W. Birkmayer and M. Wale, "Proof-of-concept model of a coherent optical beamforming network," Proc. Inst. Elect. Eng., vol. 139, no. 4, pt. J, pp. 301-304, 1992.

[5] H. Horikawa, Y. Nakasuga, and H. Ogawa, "Self-hetrodyning optical waveguide beam forming and steering network integrated on lithium niobate substrate," IEEE Trans. Microwave Theory Tech., vol. 43, pp. 2395-2401, Sept. 1995.

[6] L. B. Soldano and E. C. M. Pennings, "Optical multi-mode interference devices based on self-imaging: Principles and applications," $J$. Lightwave Technol., vol. 13, pp. 615-627, Apr. 1995.

[7] J. Stulemeijer, X. J. M. Leijtens, I. Moerman, and M. K. Smit, "Photonic ic for electronic radar beam control," in Proc. IEEE/LEOS Symp. Benelux Chapter, Eindoven, The Netherlands, Nov. 1997, pp. 205-208,

[8] T. Uiterdijk, "Integrated electro-optical switches on InP," Ph.D. dissertation, Delft Univ. of Technology, Delft, The Netherlands, 1997. 